\title{
Mouse miRNA-709 directly regulates miRNA-15a/16-1 biogenesis at the posttranscriptional level in the nucleus: evidence for a microRNA hierarchy system
}

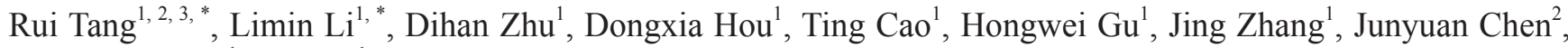
Chen-Yu Zhang ${ }^{1}$, Ke Zen ${ }^{1}$

${ }^{1}$ Jiangsu Engineering Research Center for microRNA Biology and Biotechnology, State Key Laboratory of Pharmaceutical Biotechnology, School of Life Sciences, Nanjing University, 22 Hankou Road, Nanjing, Jiangsu 210093, China; ${ }^{2}$ Marine Biology Lab, School of Life Sciences, Nanjing University, 22 Hankou Road, Nanjing, Jiangsu 210093, China

MicroRNAs (miRNAs) are endogenous noncoding RNAs ( $22 \mathrm{nt})$ that regulate target gene expression at the posttranscriptional level in the cytoplasm. Recent discoveries of the presence of miRNAs and miRNA function-required argonaute family proteins in the cell nucleus have prompted us to hypothesize that miRNAs may also have regulatory functions in the cell nucleus. In this study, we demonstrate that mouse miR-709 is predominantly located in the nucleus of various cell types and that its nuclear localization pattern rapidly changes upon apoptotic stimuli. In the cell nucleus, miR-709 directly binds to a 19-nt miR-709 recognition element on pri-miR-15a/16-1 and prevents its processing into pre-miR-15a/16-1, leading to a suppression of miR-15a/16-1 maturation. Furthermore, nuclear miR709 participates in the regulation of cell apoptosis through the miR-15a/16-1 pathway. In summary, the present study provides the first evidence that one miRNA can control the biogenesis of other miRNAs by directly targeting their primary transcripts in the nucleus.

Keywords: microRNA; nucleus; apoptosis

Cell Research (2012) 22:504-515. doi:10.1038/cr.2011.137; published online 23 August 2011

\section{Introduction}

MicroRNAs (miRNAs) are noncoding endogenous RNA molecules (18-25 $\mathrm{nt}$ ) that have emerged as a new class of gene regulators at the post-transcriptional level $[1,2]$. Previous studies $[1,3]$ have established a model for the biogenesis and function of miRNAs. The miRNA gene is first transcribed by RNA polymerase II or RNA polymerase III into primary miRNA transcript (pri-miR$\mathrm{NA}$ ), and the pri-miRNA is then cleaved into precursor

\footnotetext{
*These two authors contributed equally to this work.

Correspondence: Ke Zen ${ }^{\mathrm{a}}$, Chen-Yu Zhang ${ }^{\mathrm{b}}$, Junyuan Chen ${ }^{\mathrm{c}}$

${ }^{a}$ E-mail: kzen@nju.edu.cn

${ }^{\mathrm{b} E}$ E-mail: cyzhang@nju.edu.cn

${ }^{c}$ E-mail: chenjunyuan@163.net

${ }^{3}$ Current address: Department of Physiology and Pharmacology, University of Georgia, Athens, GA 30084, USA.

Received 29 April 2011; revised 6 July 2011; accepted 11 July 2011; published online 23 August 2011
}

miRNA (pre-miRNA; 60-80 nt) by the nuclear microprocessor complex formed by Drosha and DGCR8. PremiRNA is then transported by Exportin-5 in a RanGTPdependent manner into the cytoplasm where it is further cleaved into a double-stranded miRNA/miRNA* duplex by Dicer. Finally, one strand of the duplex (mature miRNA) is incorporated into the RNA-induced silencing complex (RISC), which further guides its function via translational repression or mRNA cleavage [4-6]. According to this model, mature miRNAs execute their functions mainly in the cytoplasm. However, recent studies have revealed that there is an extensive amount of mature miRNAs and core proteins of the RISC, such as argonaute (AGO) family proteins (AGO1 and AGO2), in the nucleus [7-11]. Based on these findings, we propose that mature miRNAs in the nucleus may directly target other nuclear noncoding RNAs, particularly pri-miRNA transcripts, thereby, modulating the biogenesis and function of these miRNAs. In the present study, we demonstrate that mouse miR-709 is located predominantly in 
the nucleus where it specifically binds to a 19-nt element on pri-miR-15a/16-1, thus blocking the processing of primiR-15a/16-1 to pre-miR-15a/16-1, leading to a suppression of miR-15a/16-1 biogenesis. Moreover, the results of this study suggest that nuclear miR-709 can participate in the regulation of cell apoptosis through the miR16/Bcl-2 pathway.

\section{Results}

\section{Predominant localization of miR-709 in the nucleus}

To obtain the nuclear miRNA profile, we purified the nuclei from HEK-293T cells or C57BL/6J mouse livers using a well-defined procedure [9], and the high purity of the isolated nuclear fractions was validated using critical nuclear RNA and protein markers including U6 small nuclear RNA and H2A histone protein (Supplementary information, Figure S1A and S1B). Hoechst 33258 staining also showed that purified nuclei were mostly intact with clear membrane boundaries (Supplementary information, Figure S1C). A survey of miRNA expression in mouse liver nuclei on a genome-wide scale was performed using a miRNA microarray assay. As compared to cellular miRNAs [12], nuclear miRNAs had a different expression pattern. A total of 1163 mammalian miRNAs were analyzed, and a panel of miRNAs including miR-709, miR-805, miR-690 and miR-122 was found to be enriched in the nucleus (Figure 1A). A total of 44 miRNAs with the highest nuclear expression levels were further examined by a TaqMan probe-based quantitative real-time polymerase chain reaction (qRT-PCR) assay (Supplementary information, Table S1), and the results confirmed that miR-709, miR-690 and miR-30e were highly expressed in the nucleus (Figure 1B).

Because the predominant nuclear expression of miR709 was confirmed by both microarray and qRT-PCR assays, we selected miR-709 as the nuclear-enriched miRNA for further analysis. First, we examined the nuclear expression profile of miR-709 in different tissues in $\mathrm{C} 57 \mathrm{BL} / 6 \mathrm{~J}$ mice, and the results clearly showed miR709 as a ubiquitous nuclear miRNA (Supplementary information, Figure S2). Second, we quantitatively evaluated the nuclear enrichment of miR-709 in mouse L929 cells using miR-29a, a typical cytoplasmic miRNA [9], as a negative control. As shown in Figure 1C, miR-709 was predominantly located in the nucleus with a relative abundance that is $>95 \%$, whereas miR-29a was not or only weakly detected in the nucleus $(<0.5 \%)$. A localization study in mouse L929 cells using synthetic miR-709 and miR-29a mimics further confirmed the nuclear localization of transfected miRNA mimics of miR-709 and the cytoplasmic localization of miR-29a (Figure 1D). In addition, synthetic pre-miR-709 and anti-miR-709 antisense oligonucleotides (ASOs) effectively impacted the nuclear miR-709 levels in mouse L929 cells and human HEK-293T cells (Supplementary information, Figure S3).

miR-709 negatively regulates $m i R-15 a$ and $m i R-16-1$ in mice

To test the hypothesis that nuclear miR-709 may directly affect the biogenesis of other miRNAs, we applied miRNA microarray assays to analyze the expression differences of miRNAs in cells with overexpressed or depleted miR-709. Among the 600 mouse miRNAs analyzed, miR-16-1 had the most significant upregulation in cells when miR-709 was depleted (Figure 2A). In agreement with these results, miR-16-1 was downregulated with the overexpression of miR-709 (Supplementary information, Figure S4).

Computational analysis further suggested that a putative 19-nt binding site for miR-709 exists on mouse primiR-15a/16-1 and that it is $\sim 0.8 \mathrm{~kb}$ downstream of the miR-15a/16-1 gene loci (Figure 2B). The minimum free energy $(\Delta \mathrm{G})$ predicted for hybridization of miR-709 with pri-miR-15a/16-1 at this site is $-41.5 \mathrm{kcal} / \mathrm{mol}$ as determined by RNA hybrid analysis [13]. Interestingly, in addition to miR-15a/16-1, the miR-16 gene has an additional copy in the mouse genome, which is called miR$15 \mathrm{~b} / 16-2$, but the expression levels of these genes are significantly lower than miR-15a [14] and do not significantly contribute to the overall population of miR-16 [15]. We searched for putative miR-709-binding sites on primiR-15b/16-2, but we did not find any hits according to our target prediction algorithm. In agreement with miR709 being a mouse-specific miRNA, the putative binding site of miR-709 was not found on human pri-miR15a/16-1. These findings suggest that miR-709 may affect miR-15a/16-1 in mice but that it may not affect miR15b/miR-16-2 in mice or miR-15a/miR-16-1 in humans.

An inverse correlation between the nuclear miR-709 level and pri-miR-15a/16-1 processing efficiency was detected among different mouse tissues. The transcriptional activity of the miR-15a/16-1 cluster in different mouse tissues was indicated by the relative expression level of pri-miR-15a/16-1. Figure $2 \mathrm{C}$ shows that although the transcriptional activity of pri-miR-15a/16-1 varied among different tissues, the expression level of mature miR-16 remained generally the same. The Pearson's correlation coefficient between the transcriptional activity of pri-miR-15a/16-1 and level of mature miR-16 was close to $0(R=-0.077)$, further suggesting a strong post-transcriptional regulation during miR-16 maturation (Figure $2 \mathrm{C})$. When we normalized mature miR-16 levels in dif- 

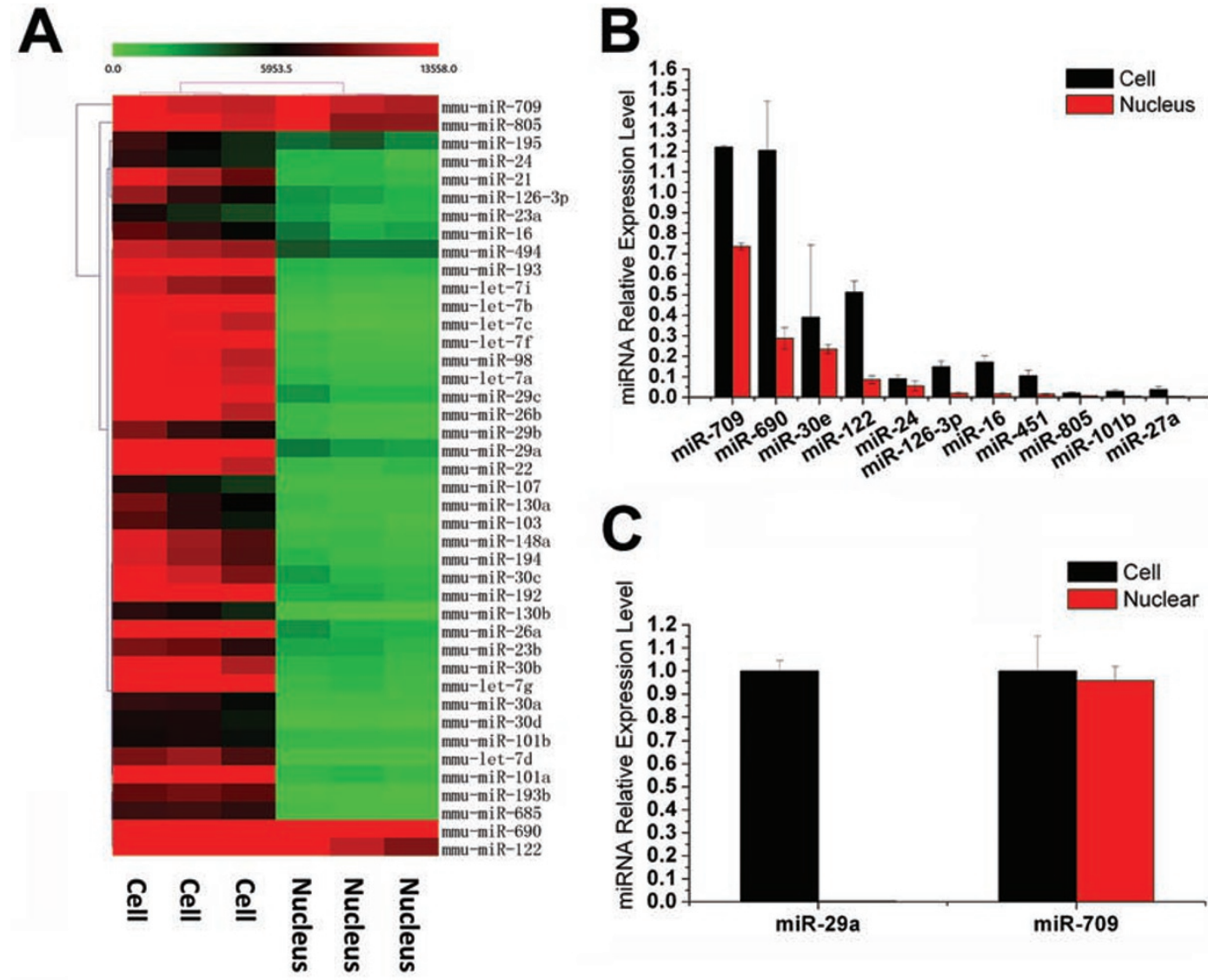

D
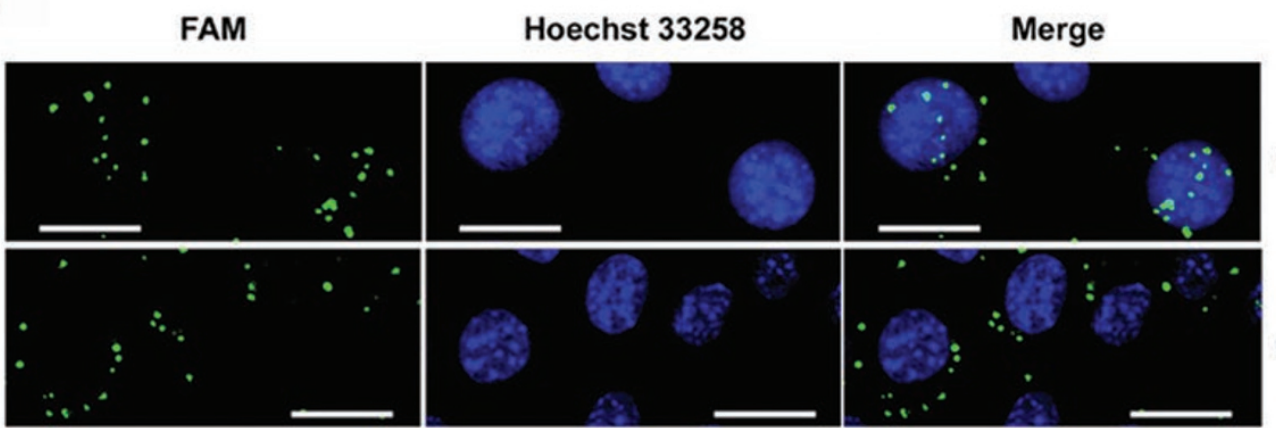

miR-709

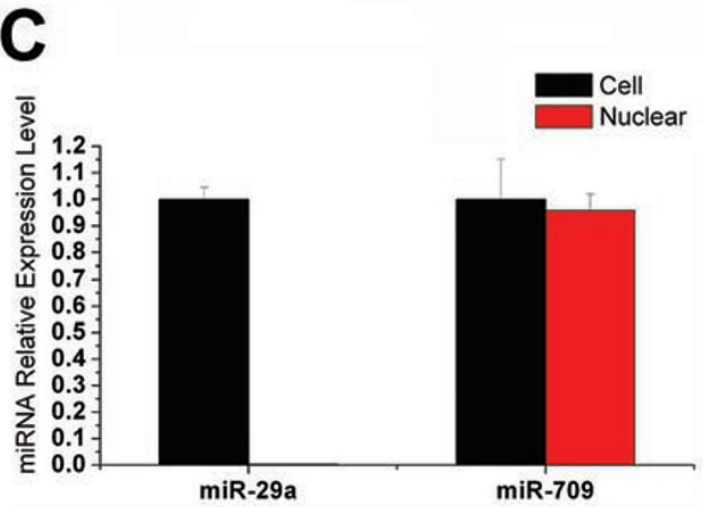

Figure 1 Predominant localization of miR-709 in the cell nucleus. (A) Nuclear miRNA expression profile in C57BL/6J mouse liver as detected by miRNA microarray. Relative expression levels for the 50 miRNAs that exhibited a signal stronger than 2000 detected on the microarray chip are shown in six columns corresponding to two groups of samples (cells and nuclei). Colors indicate relative signal intensity. The miRNA expression profile was sorted using a hierarchical clustering method (Cluster 3.0 software and Java TreeView). MiR-709 was specifically enriched in the nucleus. (B) qRT-PCR validation of 44 nuclear miRNA candidates in C57BL/6J mouse liver (only top 11 miRNAs are shown). (C) Nuclear miR-709 expression in mouse L929 cells. miR-709 predominantly localized to the nucleus with a relative nuclear abundance $>95 \%$ as compared to miR-29a (less than $0.5 \%$ nuclear abundance). (D) Nuclear localization of synthetic miR-709 mimics. The 5'-FAM-labeled miR709 mimic predominantly localized at the cell nuclei of L929 cells, and the 5'-FAM-labeled miR-29a did not. The bar represents $20 \mu \mathrm{m}$.

ferent tissues to the transcriptional activity of its gene (termed processing efficiency in Figure 2D), a modest inverse correlation existed between the nuclear miR-709 level and pri-miR-15a/16-1 processing efficiency with an $R$ value of -0.46 . If the liver was excluded, which has an extremely high nuclear miR-709 level, the Pearson's cor- 

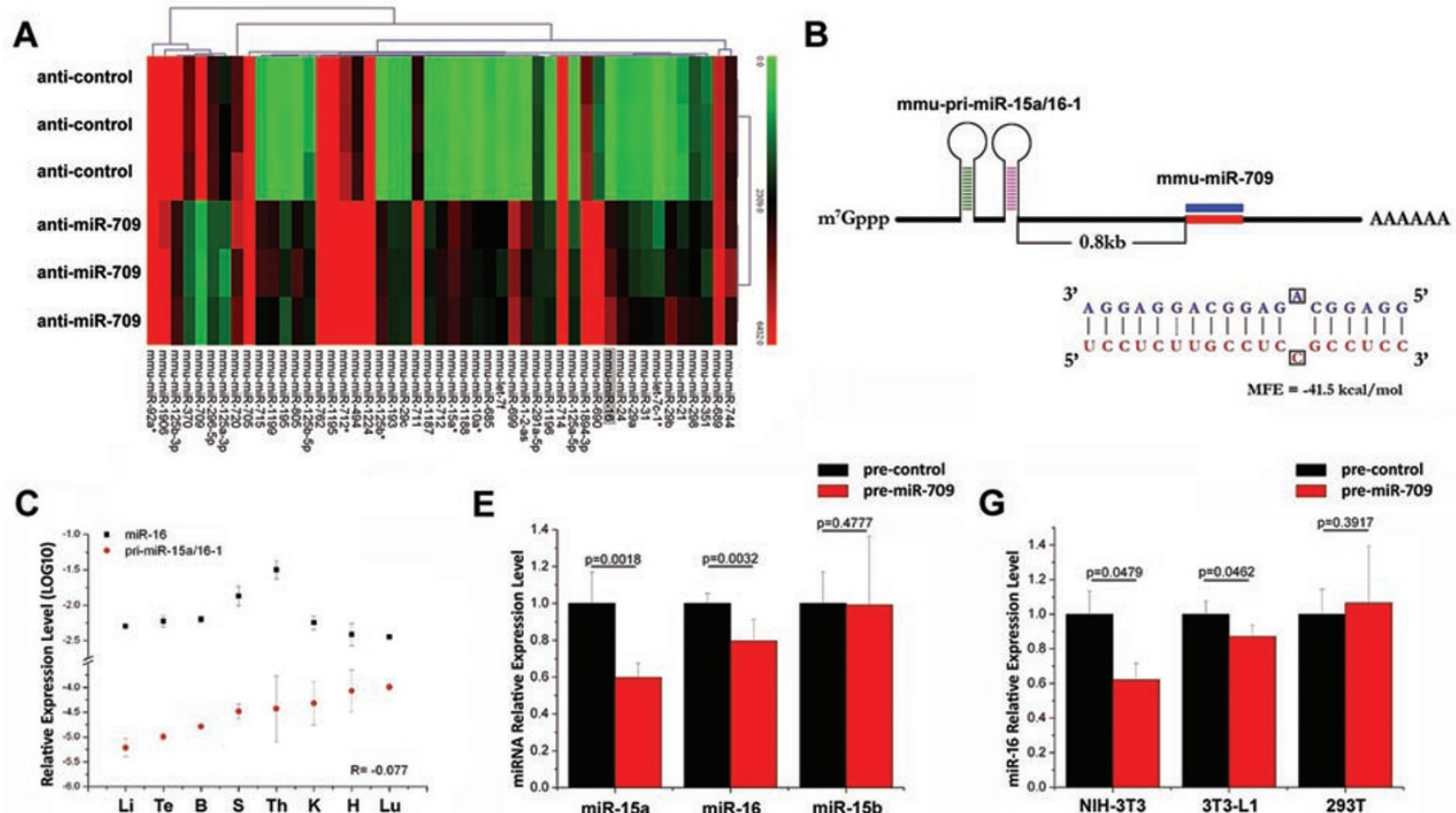

E
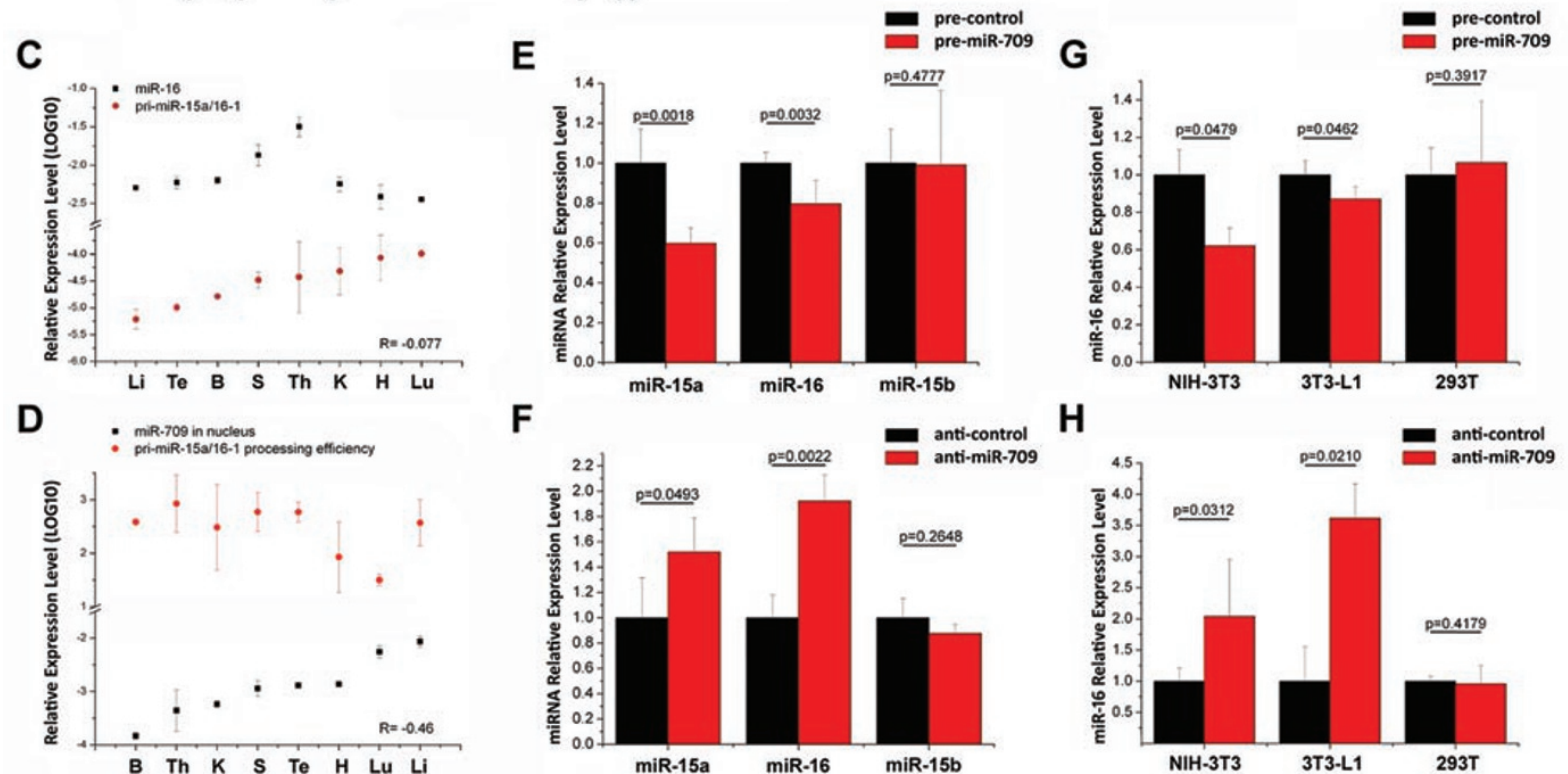

$\mathbf{F}$

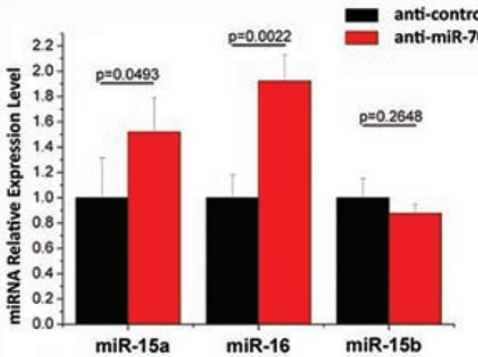

H

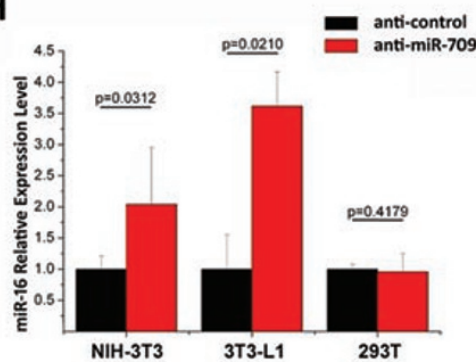

Figure 2 miR-709 negatively regulates the expression of mature miR-15a/16-1 in mice. (A) Screening for target miRNAs of miR-709 via a microarray assay. MiR-16-1 had the most significant upregulation in cells after depleting miR-709. (B) Putative binding site for miR-709 on mouse pri-miR-15a/16-1 as predicted using RNAhybrid (http://bibiserv.techfak.uni-bielefeld. de/rnahybrid). A near-perfect matching site (red rectangle) for miR-709 (blue rectangle) was located $0.8 \mathrm{~kb}$ downstream of the miR-15a/16-1 gene loci. The following abbreviation is used: MFE, minimum free energy. (C) pri-miR-15a/16-1 processing efficiency. The Pearson's correlation between relative expression levels of miR-16-1 and pri-miR-15a/16-1 was close to 0 $(R=0.006)$ in different mouse tissues including the liver $(\mathrm{Li})$, testis $(\mathrm{Te})$, brain $(\mathrm{B})$, spleen $(\mathrm{S})$, thymus $(\mathrm{Th})$, kidney $(\mathrm{K})$, heart (H) and lung (Lu). (D) Correlation between nuclear miR-709 and pri-miR-15a/16-1 processing efficiency in mice. (E, F) Relative expression levels of miR-15a, miR-16-1 and miR-15b in cells transfected with pre-miR-709 (pre-709) (E) or anti-miR-709 (anti-709) ASOs (F). At $24 \mathrm{~h}$ post-transfection, levels of miR-15a and miR-16-1 were decreased by miR-709 in L929 cells, and no significant change in the level of miR-15b was observed. (G, H) Differential regulatory effects of miR-709 on miR-16-1 in human and mouse cells transfected with pre-miR-709 (F) or anti-miR-709 ASOs (G). MiR-709-mediated miR-16-1 regulation occurred exclusively in mouse cell lines (L929, NIH-3T3 and 3T3-L1) and not in human cell lines (HEK-293T).

relation coefficient between the nuclear miR-709 level and pri-miR-15a/16-1 processing efficiency was approximately -0.67 (Supplementary information, Figure S5). These analyses strongly suggest a negative regulatory role for nuclear miR-709 in pri-miR-15a/16-1 processing.

To examine if miR-709 in the nucleus regulates the levels of mature miR-15a and miR-16-1, L929 cells were transfected with pre-miR-709 or anti-miR-709 ASOs. Mature miR-15a and miR-16-1 levels were examined by qRT-PCR. Serving as a control, the expression level of miR-15b, which has no putative binding site for miR-709 in the primary transcript, was also examined. As shown in Figure 2E and 2F, levels of mature miR-15a and miR16-1 were decreased by nuclear miR-709 $24 \mathrm{~h}$ posttransfection, and no effect of miR-709 on the miR-15b 
level was observed at this time.

Because human pri-miR-15a/16-1 do not have the putative 19-nt binding site for miR-709, we predicted that miR-709-mediated regulation of human miR-15a/16-1 would not be detected. To test this hypothesis, we employed the human-derived cell line, HEK-293T, and two additional mouse cell lines, NIH-3T3 and 3T3-L1, for the experiment. The cellular levels of miR-16-1 were detected after overexpression or depletion of miR-709. In support of our prediction, the results showed that mature miR-16-1 levels were decreased by nuclear miR-709 in the two mouse cell lines and that no significant changes were detected in the human HEK-293T cells (Figure 2G and $2 \mathrm{H})$.

miR-709 prevents the processing of pri-miR-15a/16-1 into pre-miRNA in the nucleus via direct binding to primiR-15a/16-1

We next tried to identify the mechanism underlying the negative regulatory effect of nuclear miR-709 on the biogenesis of miR-15a/16-1. First, we found that miR709 had no impact on the transcriptional activity of the miR-15a/16-1 cluster. By comparing the kinetics of primiR-15a/16-1 and miR-16 levels during miR-709-mediated regulation, we found that mature miR-16-1 gradually decreased or increased following the overexpression
A
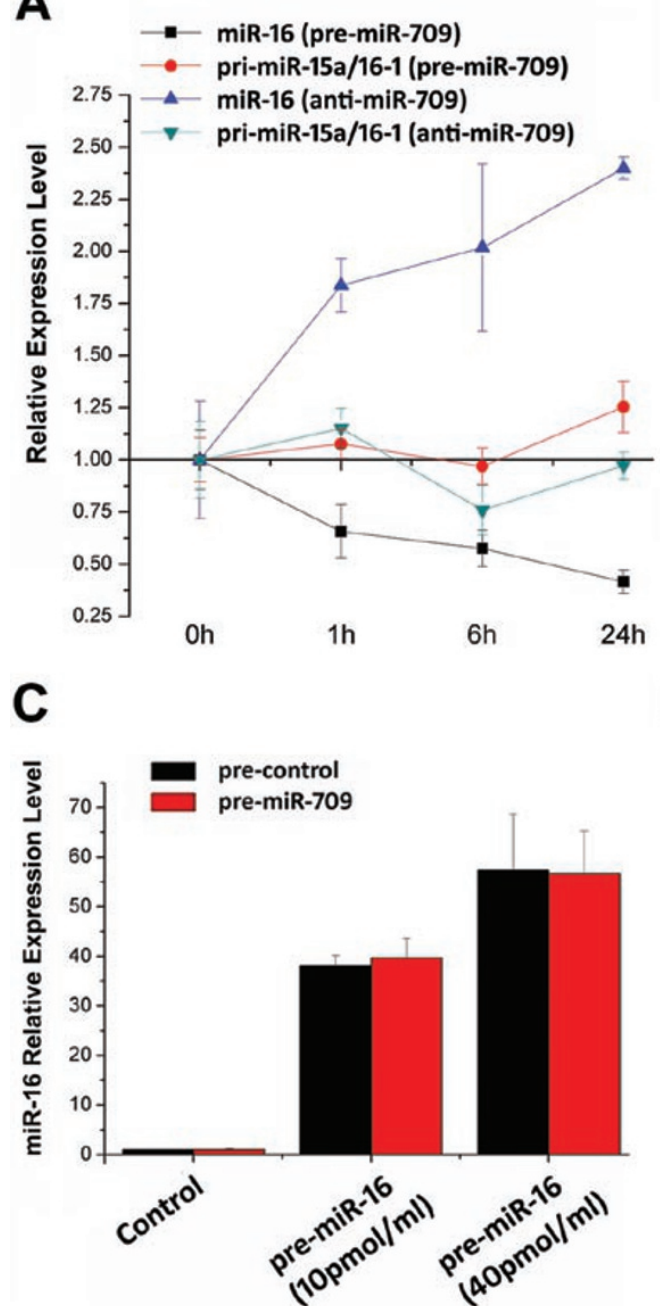

B

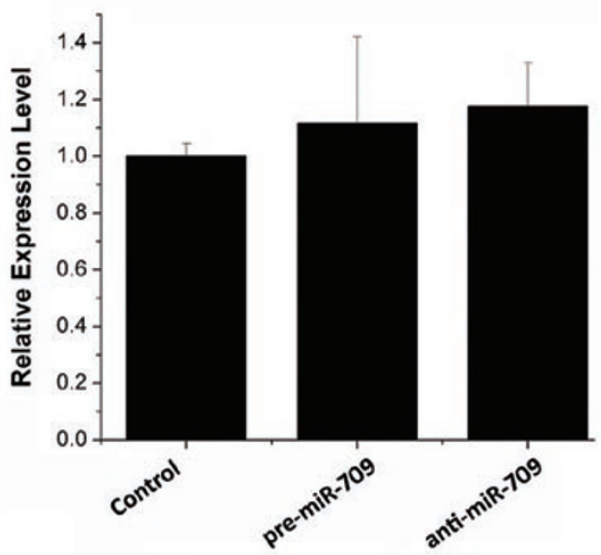

D

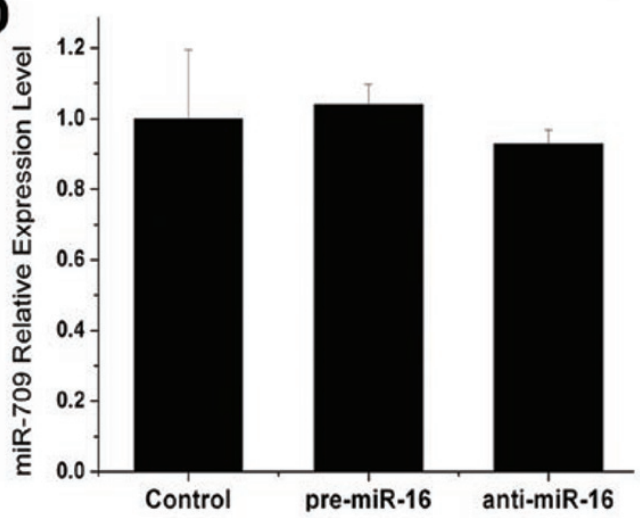

Figure 3 miR-709 suppresses the processing of pri-miR-15a/16-1 in the nucleus. (A, B) pri-miR-15a/16-1 transcriptional ratio after miR-709 overexpression or depletion. Both total cellular (0-24 h) (A) and neo-synthesized (2 h) (B) pri-miR-15a/16-1 levels remained unchanged in mouse L929 cells after miR-709 overexpression or depletion. (C) Effects of miR-709 on premiR-16-1 processing. miR-709 had no impact on pre-miR-16-1 processing in HEK-293T cells at various concentrations $(0,10$ and $40 \mathrm{pmol} / \mathrm{ml}$ ). (D) Effects of miR-16 on the cellular level of miR-709. No significant change in miR-709 levels was detected in L929 cells $24 \mathrm{~h}$ after transfection with the miR-16 precursor or ASOs. 
A

Chromosome 14qC3

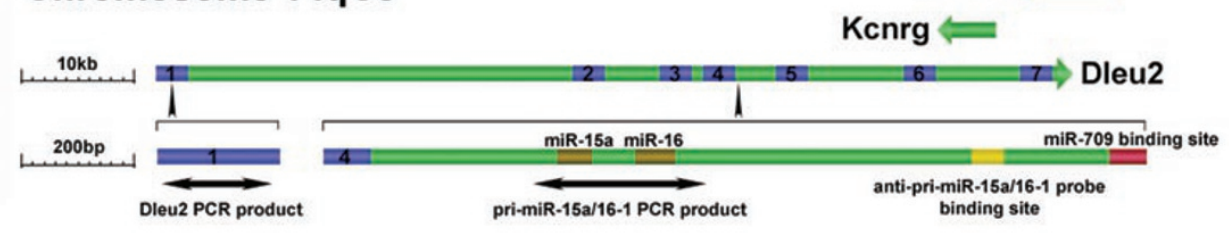

B

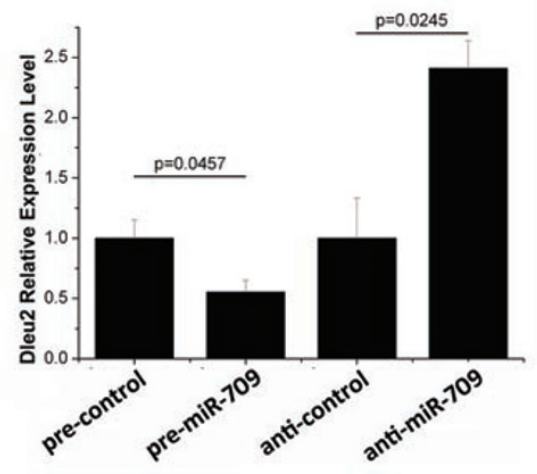

Figure 4 Nuclear miR-709 negatively impacts Dleu2 mRNA maturation efficiency in L929 cells. (A) Schematic illustration of all PCR primers and the co-precipitation of probe-binding sites on the mouse 14qC3 locus. Genes and their 5'-3' orientation are indicated by thick arrows. Exons are indicated by blue rectangles, and introns are indicated by green rectangles. (B) Impact of nuclear miR-709 on the processing of host Dleu2 mRNA.

or depletion, respectively, of nuclear miR-709 and that pri-miR-15a/16-1 remained unchanged (Figure 3A). To confirm this phenomenon, we labeled neo-synthesized pri-miR-15a/16-1 by co-transfection with biotin-16-UTP ( $50 \mu \mathrm{mol} / \mathrm{ml}$; Roche). Biotinylated RNA was purified via streptavidin-coated magnetic beads. At $2 \mathrm{~h}$ post-treatment, there were no significant differences in the levels of neo-synthesized biotin-labeled pri-miR-15a/16-1 in cells with miR-709 overexpression, miR-709 depletion or scrambled siRNA duplex overexpression (Figure 3B). Second, we applied a synthetic pre-miR-16 mimic by cotransfection with miR-709 to assess the effect of miR709 on pre-miRNA processing. As shown in Figure 3C, miR-709 had no effect on pre-miR-16 processing.

It is possible that the impact of miR-709 on mouse miR-15a/miR-16-1 may be due to general competition for the miRNA processing apparatus in the cytoplasm. To exclude this possibility, we transfected mouse L929 cells with a miR-16 precursor or antisense oligonucleotides and then examined cellular miR-709 levels. As shown in Figure 3D, there was no significant alteration of miR-709 levels among cells transfected with a miR16-1 precursor, miR-16-1 antisense oligonucleotide or scrambled siRNA duplex controls. The results suggest that the regulation of mouse miR-15a/16-1 by miR-709 is unidirectional and that the general competition for the miRNA processing apparatus in the cytoplasm does not have a major role in miR-709-mediated miRNA regulation. Given that nuclear miR-709 did not affect either the transcriptional activity of miR-15a/16-1 (Figure 3A and $3 \mathrm{~B}$ ) or the processing of pre-miR-16 (Figure $3 \mathrm{C}$ and $3 \mathrm{D}$ ), we hypothesized that nuclear miR-709 negatively regulated miR-15a/16-1 maturation at the post-transcriptional level and, more specifically, at a stage after pri-miRNA transcription but before pre-miRNA cleavage.

Moreover, because miR-15a/16-1 resides in the Dleu2 intron (Figure 4A), we also determined the effect of nuclear miR-709 on Dleu2 mRNA and found that nuclear miR-709 also had a negative effect on Dleu2 mRNA processing (Figure 4B).

To further test if the 19-nt miR-709 recognition element on mouse pri-miR-15a/16-1 was responsible for miR-709 binding and blockade of miR-15a/16-1 biogenesis, we modified the human miR-16 expression vector by inserting the 19-nt miR-709 recognition element into the miR-16-1 expression vector (Figure 5A) and then overexpressed the modified vector in human HEK-293T cells. As shown in Figure 5B, co-transfection of premiR-709 and the modified human miR-16 expression vector (pcDNA-miR-16-INS) into human HEK-293T 
A

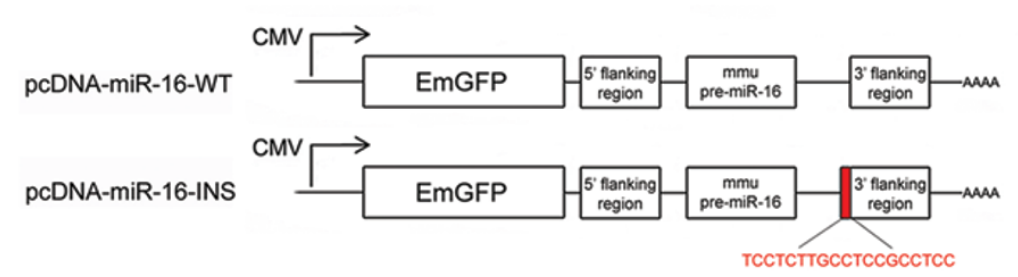

C

PMIR-luc-pri-WT (1.6kb)

PMIR-luc-pri-MUT (1.6kb)

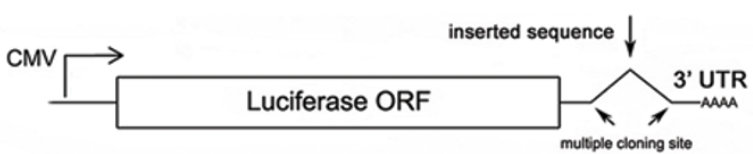

Construct map of pMIR-REPORT luciferase vector

E

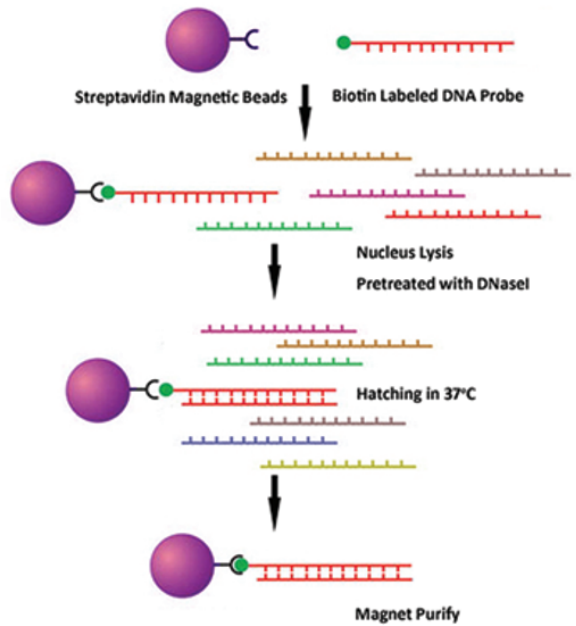

B

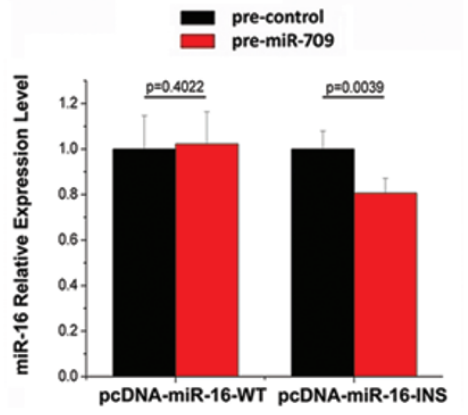

D

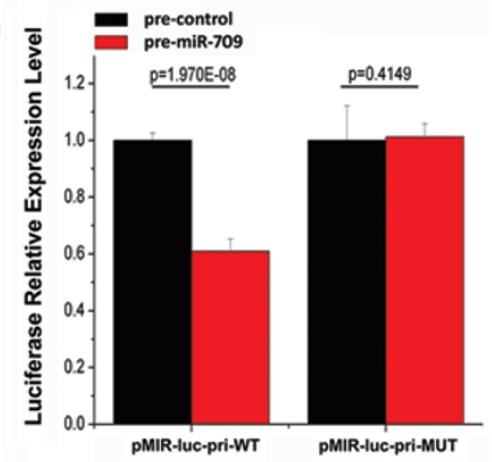

Figure 5 Direct binding of miR-709 with pri-miR-15a/16-1 in the nucleus. (A) Modification of human miR-16-1 expression vector via insertion of the 19-nt miR-709 recognition element. (B) miR-709 impaired the processing of the modified human miR-16-1 expression vector. MiR-709 did not affect the processing of wild-type miR-16 expression vector (pcDNA-miR-16WT), but it impaired the maturation of miR-16 derived from the modified miR-16 expression vector (pcDNA-miR-16-INS) in which a miR-709 binding site was inserted. (C, D) Luciferase reporter assays for the binding of mouse miR-709 to pri-miR15a/16-1. HEK-293T cells were transfected with an expression vector that contained a 2-kb sequence downstream of mouse pri-miR-15/16-1 including the miR-709 binding site (pMIR-luc-Wt) or lacking the complementary site (pMIR-luc-Mut). (E) Schematic illustration of pri-miR-15a/16-1 pull-down strategy. (F) Levels of miR-709 and pri-miR-15/16-1 (assayed by qRTPCR) in pull-down products detected by an anti-pri-miR-15a/16-1 probe, anti-mutated pri-miR-15a/16-1 probe, random probe or no probe. The following abbreviation is used: ND, non-detectable.

cells successfully induced a decrease ( $20 \%$ inhibition) in human miR-16-1 levels as compared to co-transfection of pre-miR-709 with the wild-type (WT) human miR16 expression vector (pcDNA-miR-16-WT). This result clearly demonstrated that the 19-nt miR-709 recognition element was sufficient for miR-709-mediated regulation of miR-15a/16-1 biogenesis.

A luciferase reporter assay was also employed to demonstrate the direct binding of miR-709 to pri-miR15a/16-1. In this experiment, the WT or mutant (MUT) miR-709 complementary site (CS) was cloned into a luciferase reporter plasmid (Figure 5C), and the plasmids 
A

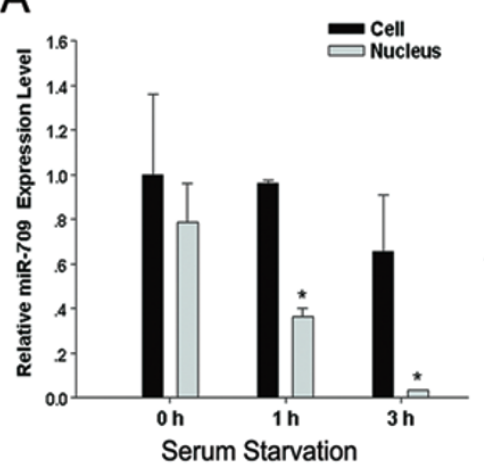

D

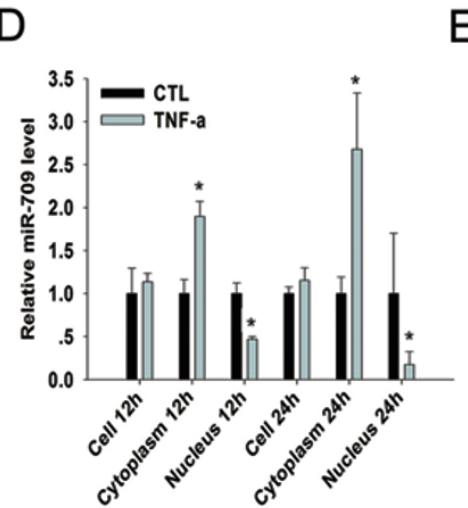

B

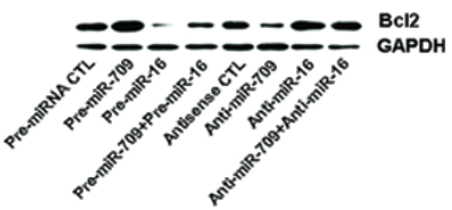

E

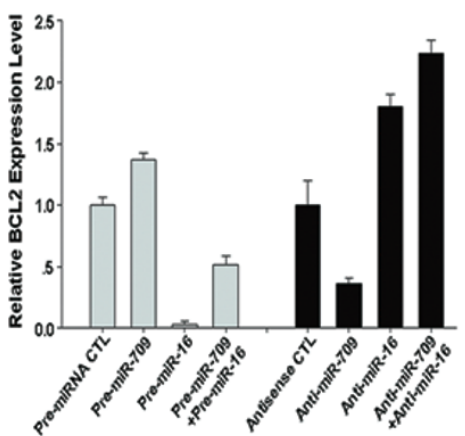

C

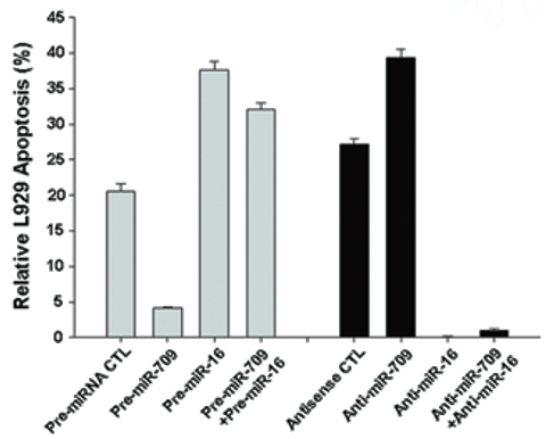

$\mathbf{F}$

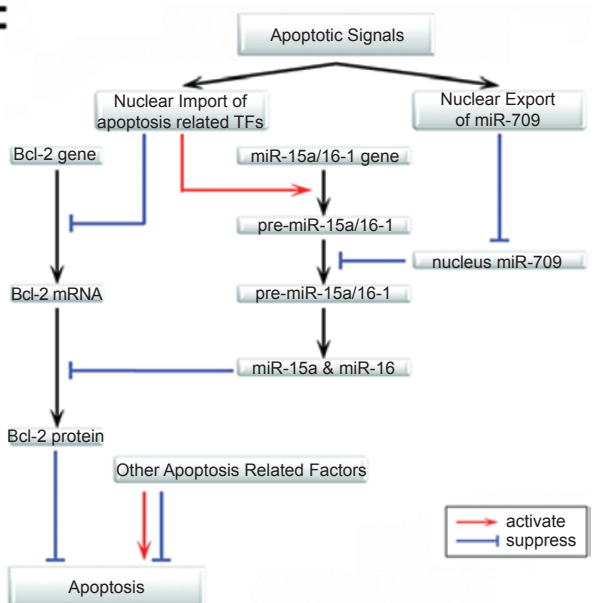

Figure 6 miR-709 regulates apoptosis through the miR-16/Bcl-2 pathway. (A) Redistribution of miR-709 in L929 cells during serum starvation-induced apoptosis. Nuclear miR-709 exited the nucleus during apoptosis, and the total cellular miR-709 levels remained unchanged. (B) miR-709 regulated Bcl-2 expression level via the miR-16 pathway. Bcl-2 protein levels in L929 cells were assessed at $24 \mathrm{~h}$ post-transfection with various oligonucleotides including pre-miRNA control, pre-miR-709, premiR-16, pre-miR-709 plus pre-miR-16, antisense control, anti-miR-709 ASOs, anti-miR-16 ASOs, and anti-miR-709 plus antimiR-16 ASOs. (C) miR-709 regulated L929 cell apoptosis through the miR-16/Bcl-2 pathway. Apoptosis was detected by PI/ Annexin $\mathrm{V}$ staining at $24 \mathrm{~h}$ post-transfection with various oligonucleotides. (D) Export of nuclear miR-709 into the cytoplasm in L929 cells during TNF- $\alpha$-induced cell apoptosis. (E) Relative Bcl-2 expression levels in TNF- $\alpha$-induced L929 cells. Cells were transfected with various oligonucleotides and then treated with TNF- $\alpha$ for $24 \mathrm{~h}$. (F) Schematic illustration of miR-709 regulation of cell apoptosis via the miR-16/Bcl-2 pathway.

were separately co-transfected with pre-miR-709 into HEK-293T cells. As expected, the WT luciferase reporter activity was significantly reduced following pre-miR-709 transfection, whereas the MUT luciferase reporter activity was unaffected (Figure 5D), suggesting that the 19-nt miR-709 recognition element is necessary for miR-709mediated regulation of miR-15a/16-1 biogenesis.

We further applied a biotin-avidin pull-down system to assess the direct binding of miR-709 to pri-miR15a/16-1. Briefly, a biotin-labeled-specific anti-primiR-15a/16-1 probe was designed and incubated with nuclear lysate from L929 cells. Pri-miR-15a/16-1 was co-precipitated via avidin-conjugated magnetic beads, and the level of miR-709 in the pull-down complex was analyzed by qRT-PCR (Figure 5E). As shown in Figure 5F, miR-709 was significantly enriched ( $\sim 10$-fold higher) in the pri-miR-15a/16-1 pull-down product as compared to the mutated pri-miR-15a/16-1 probe, random probe or no probe pull-down products, indicating that miR709 can directly bind to pri-miR-15a/16-1 in the nucleus. Interestingly, pri-miR-15a/16-1 showed almost equal enrichment as miR-709 in the pull-down products, which may implicate a one-to-one recognition ratio between these two miRNAs. Taken together, these data suggest that nuclear miR-709 can prevent the processing of primiR-15a/16-1 to pre-miRNA by directly binding to the 19-nt recognition element on pri-miR-15a/16-1. 
miR-709 participates in the regulation of cell apoptosis via the miR-15a/16-1 pathway

Because miR-15a/16-1 is a well-known miRNA cluster that induces cell apoptosis by targeting the antiapoptotic gene, Bcl-2 [16], we tested if alteration of miR$15 \mathrm{a} / 16-1$ by nuclear miR-709 had a biological role in cell apoptosis. In this experiment, we examined the expression pattern of miR-15a/16-1 during apoptosis under serum starvation conditions [17]. As expected, after a 12-h serum starvation, cellular levels of miR-16-1 and miR15 a in L929 cells were significantly upregulated (Supplementary information, Figure S6C), which correlated with a significant amount of cell apoptosis as detected by PI/Annexin V double staining [18] (Supplementary information, Figure S6A and S6B). Interestingly, a rapid downregulation of miR-709 in the nucleus was detected at an early stage following serum depletion. As shown in Figure 6A, nuclear miR-709 rapidly decreased to $50 \%$ and $5 \%$ of the original level after serum depletion for 1 and $3 \mathrm{~h}$, respectively. Surprisingly, the total cellular level of miR-709 remained largely unchanged within the same time frame. These results suggested a redistribution of miR-709, which may have been due to a rapid export of miR-709 from the nucleus to the cytoplasm under apoptotic stress. Although the mechanism for the export of mature nuclear miR-709 remains unknown, the downregulation of miR-709 in the nucleus leads to a loss of the inhibition of pri-miR-15a/16-1 processing by miR-709, which may contribute to the rapid elevation of cellular miR-15a/16-1 levels during apoptosis.

The role of nuclear miR-709 in the regulation of serum starvation-induced L929 cell apoptosis was further analyzed. As shown in Figure 6B, overexpression of miR-709 was sufficient to induce the upregulation of Bcl-2 in L929 cells. Importantly, co-expression of miR16 and miR-709 in L929 cells effectively abolished miR709-induced Bcl-2 upregulation, suggesting that Bcl-2 upregulation induced by miR-709 occurred through the downregulation of cellular miR-16. The same conclusion was drawn from the experiment in which depletion of miR-709 resulted in an elevation of miR-16 and downregulation of Bcl-2.

Downregulation of miR-709 in the nucleus and associated elevation of cellular miR-15a/16-1 were also observed during TNF- $\alpha$-induced cell apoptosis. As shown in Figure 6D, TNF- $\alpha$ treatment for 12 and $24 \mathrm{~h}$ resulted in a significant decrease in nuclear miR-709 levels but an increase in cytoplasmic miR-709 levels. However, the total cellular miR-709 level was not changed during treatment. As expected, TNF- $\alpha$ treatment caused cell apoptosis (Supplementary information, Figure S7). In cells treated with TNF- $\alpha$ for $24 \mathrm{~h}$, similar results were obtained where the overexpression of miR-709, through the downregulation of cellular miR-16, induced the upregulation of Bcl-2 (Figure 6E) and reduced cell apoptosis (Supplementary information, Figure S7). Together, these results confirmed the anti-apoptotic role of nuclear miR-709 through the miR-16/Bcl-2 pathway. As shown in Figure 6F, miR-709 protected cells from apoptosis, and the protective effect of miR-709 was abolished by increased levels of miR-16-1. Interestingly, nuclear miR709 impacted Bcl-2 expression and the cell apoptotic ratio almost to the same extent as miR-16-1, which generally executed its function in the cytoplasm.

\section{Discussion}

This study presents compelling evidence that mouse miR-709 can regulate miR-15a/16-1 biogenesis at the post-transcriptional level by directly binding to a $19-\mathrm{nt}$ recognition element on pri-miR-15a/16-1 in the nucleus. Moreover, this study demonstrates that miR-709 can participate in the regulation of cell apoptosis through modulating miR-16 maturation. This finding significantly expands the biological function of miR-709. Although miR-709 is highly expressed in various tissues and cell types during different developmental stages in mice including embryo, testes, liver, brain, ovaries, spinal cord, heart, bladder smooth muscles, cardiomyocytes and oligodendrocytes, only one validated mRNA target has been reported for this ubiquitously expressed miRNA, in which it partakes in the regulation of DNA methylation in the male germline via targeting BORIS [19]. However, based on our model illustrated in Figure 6E, miR709 , which is predominantly expressed in the nucleus, can execute its biological function by "supervising" the biogenesis of miR-15a/miR-16-1. This supervision, in turn, constitutes a short-term regulatory cascade with a quick response to apoptotic stress independent of any neo transcription. To the best of our knowledge, this is the first report on the direct miRNA-mediated regulation of biogenesis and function of miRNAs although previous studies have shown inter-miRNA antagonisms [20,21] and protein-mediated post-transcriptional control in miRNA biogenesis [22-24]. Interestingly, Tamminga et al. [19] showed that the in situ hybridization signal of miR709 is predominately located in the nucleus. Maes et al. [25] also reported that miR-709 and other proliferation essential miRNAs, such as miR-214, are significantly increased during the aging process in the liver. Both of these studies indirectly support the model in the present study. One may argue that miR-709 may directly regulate $\mathrm{Bcl}-2$ through classic miRNA/mRNA interactions instead of modulating miR-16 biogenesis. However, this 
possibility is low based on the following results: (a) miR709 elevated the level of Bcl-2 expression rather than suppressing the level of Bcl-2 expression (Figure 6); and (b) miR-709 functioned only in the nucleus and was not available for any predicted cytoplasmic mRNA targets (Figure 1).

The mechanism underlying the predominant localization of miR-709 in the nucleus remains unknown at this stage and requires further study. A previous study has reported that hexanucleotide elements may contribute to the nuclear transport of certain miRNAs, such as miR29b [9]. However, miR-709 does not have a hexanucleotide element. According to the results of the present study (Figure 1D and Supplementary information, Figure S3) showing that the identical nucleotide sequence in the miR-709 mimic was sufficient for its nucleus/cytoplasm shuttling and that changing cytoplasmic miR-709 populations impacted nuclear miR-709 populations, we suggest that there is an equilibrium between nuclear and cytoplasmic miR-709 and that the predominate nuclear localization pattern is determined by its inborn primary sequence identity. Furthermore, although miR-709 is a mouse-specific miRNA, the microarray and computational analyses performed in this study implicate that such direct miRNA/miRNA interactions may be a general phenomenon. Various miRNA targets of miR-709 other than miR-15a/16-1 were predicted in mouse revealing two highly conserved recognition patterns, which we named as "No. 6 bubble" and "No. 16 bubble", respectively (Supplementary information, Figure S8A, S8B, and Table S2).

It is generally believed that biological processes are controlled by activating a hierarchical cascade of interdependent regulators, such as transcription factors or protein kinases [26, 27]. By illustrating that a miRNA can regulate the biogenesis of other miRNAs, the results of the present study suggest that certain miRNAs can serve as a novel group of interdependent regulators at post-transcriptional level, in a so-called "miRNA hierarchy system" (Supplementary information, Figure S9). In summary, the present study demonstrates for the first time that mouse miR-709 can directly target the primary transcript of miR-15a/16-1 in the nucleus and control their biogenesis. The discovery of miRNA-mediated regulation of miRNA biogenesis provides the first evidence of a potential hierarchical structure among miRNAs.

\section{Materials and Methods}

\section{Reagents, cells and antibodies}

The mouse L929, NIH-3T3, 3T3-L1 and human HEK-293T cell lines were purchased from the China Cell Culture Center (Shanghai, China). Cells were maintained at $37{ }^{\circ} \mathrm{C}$ in a humidi- fied 5\% $\mathrm{CO}_{2}$ incubator in Dulbecco's Modified Eagle's Medium (GIBCO, CA, USA) containing 10\% fetal bovine serum (Gibco, CA, USA), 100 units $/ \mathrm{ml}$ penicillin and $100 \mu \mathrm{g} / \mathrm{ml}$ streptomycin. An anti-Bcl-2 (50E3) antibody was purchased from Cell Signaling (Danvers, MA, USA), and anti-GAPDH and anti-Histone H2A (C19) antibodies were purchased from Santa Cruz Biotechnology (CA, USA). Synthetic RNA molecules including pre-miR-709, anti-miR-709, pre-miR-16, anti-miR-16 and scrambled negative control oligonucleotides (pre-ncRNA and anti-ncRNA) were purchased from Ambion (Austin, TX, USA). Synthetic 5'-FAMlabeled miR-709 and miR-29a oligonucleotide duplexes were purchased from Invitrogen (Shanghai, China).

\section{Preparation of subcellular organelles}

For the purification of nuclei, cells (tissues were processed and filtered through a cell strainer to obtain cell suspensions) were washed three times with PBS on ice followed by centrifugation at $300 \times \mathrm{g}$ for $5 \mathrm{~min}$. Cell pellets were resuspended in lysis buffer (10 mM Tris, $\mathrm{pH} 8.0 ; 140 \mathrm{mM} \mathrm{NaCl}, 1.5 \mathrm{mM} \mathrm{MgCl}_{2}$, and $0.5 \%$ nonidet P-40), incubated on ice for $10 \mathrm{~min}$, and then centrifuged at $1000 \times g$ for $5 \mathrm{~min}$ at $4{ }^{\circ} \mathrm{C}$. Nuclear pellets were washed twice with lysis buffer followed by a final wash with nuclear wash buffer (lysis buffer containing 1\% Tween-40 and $0.5 \%$ deoxycholic acid). For liver mitochondria purification, adult male C57BL/6 mice (6-8 weeks) were fasted for $12 \mathrm{~h}$ and sacrificed by exsanguination under ether anesthesia followed by cervical dislocation. The livers were removed and rinsed in physiological saline. Approximately $3 \mathrm{~g}$ of minced liver was prepared as a $10 \%(\mathrm{wt} / \mathrm{vol})$ homogenate in an ice-cold solution of homogenate buffer containing $250 \mathrm{mM}$ sucrose and $10 \mathrm{mM}$ Tris- $\mathrm{HCl}(\mathrm{pH}$ 7.4). The homogenate was centrifuged at $700 \times \mathrm{g}$ for $10 \mathrm{~min}$ at $4{ }^{\circ} \mathrm{C}$ with $340 \mathrm{mM}$ sucrose and $10 \mathrm{mM}$ Tris- $\mathrm{HCl}$ ( $\mathrm{pH}$ 7.4). The supernatant was centrifuged for 10 min at $10000 \times g$, and the mitochondrial pellet was then washed twice under the same conditions using EGTA- and EDTA-depleted wash buffer.

Transfection of cells with ncRNA, pre-miRNA or anti-miRNA

Cells were seeded in 6-well plates or 10-mm dishes, and they were transfected the following day using Lipofectamine 2000 (Invitrogen) according to the manufacturer's instructions. For the transfection, 20 pmol RNA or 5 pmol plasmid DNA per $10^{5}$ cells was used. Cells were harvested $24 \mathrm{~h}$ after transfection for real-time PCR analysis and western blotting.

\section{microRNA microarray}

Total RNA $(50 \mu \mathrm{g})$ was purified using the mirVANA miRNA isolation kit (Ambion, Inc.) to enrich the small RNA fraction. Purified RNA was labeled with fluorescein, and hybridization was performed on a miRNA microarray chip (miRNA microarray V4.0, CapitalBio Corp., Beijing, China) containing 1320 probes in triplicate corresponding to 988 human, 627 mouse and 350 miRNA genes. Finally, hybridization signals were detected, and scanner images were quantified.

\section{RNA isolation and qRT-PCR of mature miRNAs}

Total RNA was extracted from cells or tissues using Trizol reagent (Invitrogen). qRT-PCR was carried out using TaqMan miRNA probes (Applied Biosystems, Foster City, CA, USA) according to the manufacturer's instructions. Briefly, total RNA was reverse transcribed to cDNA using M-MLV reverse transcriptase (Promega) 
and a stem-loop RT primer (Applied Biosystems). Real-time PCR was performed using a TaqMan PCR kit and an Applied Biosystems 7900 Sequence Detection System (Applied Biosystems). All reactions including no template controls were run in triplicate. After the reaction, the $\mathrm{C}_{\mathrm{T}}$ values were determined using fixed threshold settings. miRNA expression in the cells or nuclei was normalized to U6 snRNA, and mRNA expression in the cells was normalized to $\beta$-actin. The following primer sequences were used: pri-15a/16-1 forward,TCTGTAAATTACTATTGAGGTGCTAGG AGT; pri-15a/16-1 reverse,TACAGTTGTAGAGTATTGCCAAC CTTACTT; U6 forward, CTCGCTTCGGCAGCACA; U6 reverse, AACGCTTCACGAATTTGCGT; Dleu2 forward, GCAGACCGGCCTAGGGGACA; Dleu2 reverse, AGCGACGGAGGAAGACCCCC; $\beta$-actin forward, GAGACCTTCAACACCCCAGC; and $\beta$-actin reverse, ATGTCACGC ACGATTTCCC.

\section{Pri-miR-15a/16-1 pull-down assay}

Briefly, a DNA probe complementary to mouse pri-miR15a/16-1 was synthesized, labeled with biotin at both $5^{\prime}$ and $3^{\prime}$ terminals and dissolved in $500 \mu \mathrm{l}$ of wash/binding buffer $(0.5$ $\mathrm{M} \mathrm{NaCl} ; 20 \mathrm{mM}$ Tris- $\mathrm{HCl}, \mathrm{pH} 7.5$; and $1 \mathrm{mM}$ EDTA) at a final concentration of $8 \mathrm{pmol} / \mu \mathrm{l}$. The probe was then incubated with streptavidin-coated magnetic beads (New England BioLabs, Cat. $\mathrm{S} 1420 \mathrm{~S})$ at $25^{\circ} \mathrm{C}$ for $1 \mathrm{~h}$ to generate probe-coated magnetic beads. Purified nuclear lysate was pretreated with DNase I (Takara, Cat. D2210) and then incubated with probe-coated beads at $37^{\circ} \mathrm{C}$ for $3 \mathrm{~h}$. After washing four times with the wash/binding buffer, a magnet was applied to cause the beads/RNA complex to bind to the side of the tube. RNA was eluted from the streptavidin beads with $10 \mathrm{mM}$ EDTA ( $\mathrm{pH} 8.2$ ) by incubation at $90{ }^{\circ} \mathrm{C}$ for $10 \mathrm{~min}$, and the RNA was analyzed by qRT-PCR. The following primer sequences were used: anti-pri-miR-15a/16-1 pull-down probe, TCATGGCAGCCTGGTCTACATGG; and random pull-down probe, TGATGTCTAGCGCTT GGGCTTTG.

\section{Western blot analysis}

Samples of tissues and cultured cells were lysed in a buffer (50 $\mathrm{mM}$ Tris- $\mathrm{HCl}, \mathrm{pH} 7.4 ; 150 \mathrm{mM} \mathrm{NaCl} ; 1 \% \mathrm{NP}-40$; and $0.1 \% \mathrm{SDS}$ ), sonicated $(6 \times 1.5 \mathrm{sec}, 30 \%$ power $)$ and centrifuged at $12000 \times$ $g$ for $10 \mathrm{~min}$ at $4{ }^{\circ} \mathrm{C}$. The supernatant fraction was removed, and the protein concentration was determined by a BCA assay (Pierce, Rockford, USA). Aliquots of proteins $(60-100 \mu \mathrm{g})$ were separated on $10 \%$ sodium dodecyl sulfate-polyacrylamide gels (SDS-PAGE) and transferred to polyvinylidene difluoride membranes. The membranes were blocked for $1 \mathrm{~h}$ at room temperature with $5 \%$ non-fat milk in Tris-buffered saline (TBS) plus Tween-20 (TBST) followed by an overnight incubation at $4{ }^{\circ} \mathrm{C}$ with antibodies diluted in blocking buffer. After three 10-min washes with TBST, blots were incubated at room temperature for $1 \mathrm{~h}$ with the appropriate secondary antibody conjugated to horseradish peroxidase, and protein expression was detected with an enhanced chemiluminescent reagent (Cell Signaling). The autoradiographic intensity of each band was scanned and quantified using BandScan software (Glyko Inc.).

\section{Plasmid construction}

To construct pcDNA-miR-16-WT, a 93-bp sequence of premiR-16-1 was amplified by PCR using mouse genomic DNA as a template. The PCR product was inserted into the pcDNA6.2-GW/
EmGFP-miR plasmid (Invitrogen). To construct pMIR-luc-pri$\mathrm{WT}$, a 1.6-kb sequence downstream of mouse pri-miR-15a/16-1 was amplified by PCR using mouse genomic DNA as a template. The PCR product was inserted into the pMIR-report plasmid (Ambion). Efficient insertion was confirmed by sequencing.

\section{Luciferase reporter assay}

For luciferase reporter assays, $0.2 \mu \mathrm{g}$ of firefly luciferase reporter plasmid, $0.1 \mu \mathrm{g}$ of $\beta$-galactosidase expression vector (Ambion) and equal amounts (20 pmol) of pre-miR-709 or scrambled negative control RNA were transfected into cells plated in 24-well plates. The $\beta$-galactosidase vector was used as a transfection control. At $24 \mathrm{~h}$ post-transfection, cells were assayed using luciferase assay kits (Promega).

\section{Statistical analysis}

Real-time PCR was performed in triplicate, and each experiment was repeated at least three times. Data were presented as the means \pm SD of at least three independent experiments, and differences were considered statistically significant at $P<0.05$ using Student's $t$-test.

\section{Acknowledgments}

This work was supported by grants from the National Natural Science Foundation of China (30871019, 30890044 and 30988003 ) and the National Basic Research Program of China (2007CB815804 and 2011CB504803863).

\section{References}

1 Bartel DP. MicroRNAs: genomics, biogenesis, mechanism, and function. Cell 2004; 116:281-297.

2 Ambros V. The functions of animal microRNAs. Nature 2004; 431:350-355.

3 Kim VN. MicroRNA precursors in motion: exportin-5 mediates their nuclear export. Trends Cell Biol 2004; 14:156-159.

4 Lewis BP, Burge CB, Bartel DP. Conserved seed pairing, often flanked by adenosines, indicates that thousands of human genes are microRNA targets. Cell 2005; 120:15-20.

5 Schnall-Levin M, Zhao Y, Perrimon N, Berger B. Conserved microRNA targeting in Drosophila is as widespread in coding regions as in 3'UTRs. Proc Natl Acad Sci USA 2010; 107:15751-15756.

6 Shin C, Nam JW, Farh KK, Chiang HR, Shkumatava A, Bartel DP. Expanding the microRNA targeting code: functional sites with centered pairing. Mol Cell 2010; 38:789-802.

7 Meister G, Landthaler M, Patkaniowska A, Dorsett Y, Teng G, Tuschl T. Human Argonaute2 mediates RNA cleavage targeted by miRNAs and siRNAs. Mol Cell 2004; 15:185-197.

8 Ritland Politz J, Zhang F, Pederson T. MicroRNA-206 colocalizes with ribosome-rich regions in both the nucleolus and cytoplasm of rat myogenic cells. Proc Natl Acad Sci USA 2006; 103:18957.

9 Hwang HW, Wentzel EA, Mendell JT. A hexanucleotide element directs microRNA nuclear import. Science 2007; 315:97-100.

10 Ritland Politz J, Hogan E, Pederson T. MicroRNAs with a nucleolar location. RNA 2009; 15:1705. 
11 Ohrt T, Mutze J, Staroske W, et al. Fluorescence correlation spectroscopy and fluorescence cross-correlation spectroscopy reveal the cytoplasmic origination of loaded nuclear RISC in vivo in human cells. Nucleic Acids Res 2008; 36:6439-6449.

12 Bian Z, Li LM, Tang R, et al. Identification of mouse liver mitochondria-associated miRNAs and their potential biological functions. Cell Res 2010; 20:1076-1078.

13 Rehmsmeier M, Steffen P, Hochsmann M, Giegerich R. Fast and effective prediction of microRNA/target duplexes. $R N A$ 2004; 10:1507-1517.

14 Landgraf P, Rusu M, Sheridan R, et al. A mammalian microRNA expression atlas based on small RNA library sequencing. Cell 2007; 129:1401-1414.

15 Calin GA, Liu CG, Sevignani C, et al. MicroRNA profiling reveals distinct signatures in B cell chronic lymphocytic leukemias. Proc Natl Acad Sci USA 2004; 101:11755-11760.

16 Cimmino A, Calin GA, Fabbri M, et al. miR-15 and miR-16 induce apoptosis by targeting BCL2. Proc Natl Acad Sci USA 2005; 102:13944-13949.

17 Wagner A, Small M, Hay N. Myc-mediated apoptosis is blocked by ectopic expression of Bcl-2. Mol Cell Biol 1993; 13:2432.

18 Bian Z, Li L, Cui J, et al. Role of miR-150-targeting c-Myb in colonic epithelial disruption during dextran sulphate sodiuminduced murine experimental colitis and human ulcerative colitis. J Pathol 2011; 225:544-553.

19 Tamminga J, Kathiria P, Koturbash I, Kovalchuk O. DNA damage-induced upregulation of miR-709 in the germline downregulates BORIS to counteract aberrant DNA hypom- ethylation. Cell Cycle 2008; 7:3731.

20 Forrest A, Kanamori-Katayama M, Tomaru Y, et al. Induction of microRNAs, mir-155, mir-222, mir-424 and mir-503, promotes monocytic differentiation through combinatorial regulation. Leukemia 2010; 24:460-466.

21 Yu J, Ryan D, Getsios S, Oliveira-Fernandes M, Fatima A, Lavker R. MicroRNA-184 antagonizes microRNA-205 to maintain SHIP2 levels in epithelia. Proc Natl Acad Sci USA 2008; 105:19300.

22 Luciano D, Mirsky H, Vendetti N, Maas S. RNA editing of a miRNA precursor. $R N A$ 2004; 10:1174.

23 Yang W, Chendrimada T, Wang Q, et al. Modulation of microRNA processing and expression through RNA editing by ADAR deaminases. Nat Struct Mol Biol 2005; 13:13-21.

24 Wulczyn FG, Smirnova L, Rybak A, et al. Post-transcriptional regulation of the let-7 microRNA during neural cell specification. FASEB J 2007; 21:415-426.

25 Maes OC, An J, Sarojini H, Wang E. Murine microRNAs implicated in liver functions and aging process. Mech Ageing Dev 2008; 129:534-541.

26 Stollewerk A, Schoppmeier M, Damen WG. Involvement of Notch and Delta genes in spider segmentation. Nature 2003; 423:863-865.

27 Luo W, Wickramasinghe SR, Savitt JM, Griffin JW, Dawson TM, Ginty DD. A hierarchical NGF signaling cascade controls Ret-dependent and Ret-independent events during development of nonpeptidergic DRG neurons. Neuron 2007; 54:739-754.

(Supplementary information is linked to the online version of the paper on the Cell Research website.) 